\title{
Selegiline Effects on Cocaine-Induced Changes in Medial Temporal Lobe Metabolism and Subjective Ratings of Euphoria
}

George Bartzokis, M.D., Mace Beckson, M.D., Thomas Newton, M.D., Mark Mandelkern, M.D., Ph.D., Jim Mintz, Ph.D., Jennifer A. Foster, B.S., Walter Ling, and T. Peter Bridge, M.D.

To test the effect of selegiline, a specific monoamine oxidase$B(M A O-B)$ inhibitor, on the cerebral metabolic and euphorigenic effects of cocaine in experienced users, eight cocaine-dependent (CD) subjects were evaluated using a within-subjects design. Each subject participated in two pairs of [F-18]-fluorodeoxyglucose (FDG)-positron emission tomography (PET) scans (baseline scan followed $24 \mathrm{~h}$ later by a second scan obtained in conjunction with a 40-mg cocaine infusion) performed before and after a 1-week period of daily treatment with $10 \mathrm{mg}$ selegiline administered orally. The hippocampus and amygdala were evaluated because of their hypothesized involvement in the addiction process, and the thalamus was evaluated as a comparison region. Following 7 days of selegiline treatment, the magnitude of the subjective euphoria ("high") produced by cocaine infusion was reduced by $40 \%$ (cocaine by selegiline interaction $F=7.15, d f=1,21, p=$ .014). Selegiline treatment also altered glucose utilization (normalized against whole brain counts) in the two limbic regions, but not the thalamus. In the amygdala, the effects of cocaine differed, depending upon whether or not patients were being treated with selegiline (cocaine by selegiline interaction $F=4.67, d f=1,19.8, \mathrm{p}=.043)$. A different effect was observed in the hippocampus, where selegiline treatment decreased metabolic activity irrespective of whether cocaine was given (main effect $F=7.70, d f=1,20$, $\mathrm{p}=.012$ ). The concomitant changes in both the subjective experience of the "high" and normalized amygdala glucose utilization after selegiline treatment, suggest that a relationship exists between cocaine-induced euphoria and limbic metabolism. The data suggest that selegiline may be a useful adjunct in the treatment of cocaine dependence.

[Neuropsychopharmacology 20:582-590, 1999]

(C) 1999 American College of Neuropsychopharmacology. Published by Elsevier Science Inc.
From the Psychiatry Service (GB), Little Rock VA Medical Center, Little Rock, Arkansas, 72114; Department of Psychiatry (GB), University of Arkansas for Medical Sciences, Little Rock, Arkansas, 72205; Psychiatry Service (GB, MB, TN, JM, WL), West Los Angeles VA Medical Center, Los Angeles, California, 90073; Research Service (JAF), West Los Angeles VA Medical Center, Los Angeles, California, 90073; Department of Psychiatry (MB, TN, JM, WL), University of California, Los Angeles, California, 90074; Nuclear Medicine Service (MM), West Los Angeles VA Medical Center, Los Angeles, California 90073; Department of Physics (MM), University of California, Irvine, California, 92697; and Medications Development Division (TPB), National Institute on Drug Abuse, Rockville, Maryland, 20857.

Address correspondence to: George Bartzokis, M.D., North Little Rock VA Medical Center (116A/NLR), 2200 Fort Roots Drive, Bldg. 170, North Little Rock, AR 72114
KEY WORDS: Cocaine; Selegiline; PET; MRI; Hippocampus; Amygdala; Euphoria

Most screening of medications for the treatment of human addiction is based on animal models. Psychostimulant dependence lacks the clear physiologic dependence syndrome seen in opiate and alcohol dependence; therefore, animal models of psychostimulant dependence evaluate behavioral parameters. However, the

Presented in part at the 36th Annual Meeting of the American College of Neuropsychopharmacology.

Received March 17, 1998; revised July 30, 1998; accepted August 3, 1998. 
lack of subjective responses in animal models and the vast differences between the behavior of human addicts and animal models of addiction, make it desirable to screen candidate medications based upon their ability to change the subjective experiences of psychostimulant addicts (Satel et al. 1995; Berger et al. 1996).

One aspect of addiction where a pharmacological intervention might prove effective is the euphoric subjective experience to drugs that reinforces drug use behaviors (O'Brien et al. 1996; Volpicelli et al. 1995). The effects of cocaine infusion on regional brain function have been studied in only two prior studies of cocaine addicts. In the first of these, 40-mg cocaine infusion reduced cerebral glucose metabolism globally, as measured with [F-18] fluorodeoxyglucose and positron emission tomography (PET) (London et al. 1990). A subsequent single-photon emission tomography (SPECT) study of cerebral perfusion showed that, after normalizing the data to counts in the cerebellum, $48 \mathrm{mg}$ of cocaine decreased blood flow in frontal cortical and basal ganglia regions (Pearson et al. 1993).

Although our understanding of the euphoria or "high" produced by cocaine in humans is limited, animal studies indicate that mesolimbic dopamine neurotransmission and the limbic system play major roles in cocaineinduced reinforcement (for reviews see Wise 1996; White 1996). Effects on mesocorticolimbic dopaminergic pathways are believed to be critical to the development of the dependence syndrome (Koob and Weiss 1992; Schultz et al. 1993). Efforts to develop a treatment for cocaine abuse have focused on dopaminergic systems, with the assumption that effective medications must substitute for, normalize, or block the effects of cocaine on this system (Blaine and Ling 1992; Tutton and Crayton 1993). Selegiline was chosen for this protocol because of its low abuse potential (Schneider et al. 1994), its safety when given in conjunction with cocaine infusion (Haberny et al. 1995), and its indirect dopamine agonist effects. These effects consist of increasing dopamine availability by reducing dopamine breakdown through MAO-B inhibition, increasing levels of betaphenylethylamine (another MAO-B specific substrate) resulting in increased synaptic dopamine release and decreasing dopamine reuptake, and enhancing catecholaminergic neuron activity (Paterson et al. 1995; Knoll et al. 1996) (for review see Gerlach et al. 1996). Dopamine agonists with higher abuse potential have also been reported, by addicts who relapsed while being treated with these agents, to reduce the reinforcing subjective experience of euphoria produced by cocaine (Rothman 1995; Rothman et al. 1996).

Animal studies have shown that limbic structures, such as the hippocampus and amygdala, are important in the brain's response to psychostimulants (Stein and Fuller 1993; Imperato et al. 1993a; Madras and Kaufman 1994; Pontieri et al. 1995; Lyons et al. 1996; Hsu et al.
1996; Wilson et al. 1994; White 1996; Whitelaw et al. 1996), human PET studies of cue-induced cocaine craving have shown activation in the medial temporal lobe (Childress et al. 1995; Grant et al. 1996), and human functional magnetic resonance imaging studies have shown that changes in these regions are associated with subjective effects in cocaine infusion experiments (Breiter et al. 1997). In the current study, we focused on limbic structures of the medial temporal lobe (amygdala and hippocampus), and for the purpose of evaluating the specificity of the limbic findings, we also examined the thalamus as a comparison region. The thalamus was chosen because primate work indicated that acute cocaine administration had few effects on rates of glucose utilization in the thalamus (Lyons et al. 1996); unlike the limbic regions, the thalamus was not associated with subjective cravings of a "rush" in human functional imaging studies (Childress et al. 1995; Grant et al. 1996; Breiter et al. 1997).

\section{METHODS}

\section{Subjects}

Seventeen nontreatment-seeking subjects who smoked "crack" cocaine and had prior IV drug use (either cocaine or opiates) at some time in their past were recruited into the study. After complete description of the study to the subjects, written informed consent was obtained. Following administration of the first two test doses of IV cocaine, six subjects were excluded for medical reasons (cocaine-induced hypertension, arrhythmia, etc.). Of the 11 subjects evaluated with PET scans, three completed only the first two scans (excluded for illicit drug use during the study). The remaining eight subjects completed all four scans, and analyses were based on their data. The eight subjects, consisting of seven males and one female (seven African Americans and one Caucasian), had a mean age of 41.3 years (SD = 5.0 ), with a mean duration of cocaine dependence of 10.6 years $(\mathrm{SD}=5.8)$.

All subjects met DSM-IV criteria for cocaine dependence and had smoked an average of at least $1 / 2$ gram (quantified as \$50) of "crack" cocaine per week for more than 6 months (assessed by self-report and a positive urine benzoylecognine test within 2 weeks prior to entering the study). Cocaine was the primary current drug of abuse, although subjects reporting use of alcohol without meeting DSM-IV criteria for alcohol dependence were included. Females were included only if they were unable to conceive (i.e., were surgically sterilized, were using birth control pills, an intrauterine device, or consistently using condoms) to avoid possible fetal exposure to cocaine and radiation.

Subjects were excluded for: age under 21 or over 50 years; dependence on other substances of abuse except 
for tobacco; report of opiate abuse or urine positive for opiates within the past month; participation in more than two other research protocols involving cocaine administration in the past year; participation in any other research protocols involving PET scanning or any other nuclear imaging procedures within the past year; history of previous medically adverse reaction to cocaine; stroke, epilepsy or psychiatric illness other than cocaine dependence; untreated or clinically significant heart disease or hypertension; significant abnormalities detected on physical exam, laboratory examination, EKG, or EEG; positive HIV test or positive pregnancy test (for females); magnetic resonance imaging (MRI) evidence of extensive atrophy (ventricle/brain ratio $>1: 10$ ).

\section{Study Protocol}

Subjects meeting the above criteria were hospitalized 4 days before beginning the protocol, and urine tests were done to verify the absence of illicit cocaine use prior to and during the 2-week infusion protocol. Therefore, the minimum abstinence from illicit cocaine use by the time of the first PET scan would have been 1 week. Subjects received study pills in a single-blind fashion. They were told only that they were to receive either selegiline $10 \mathrm{mg}$ or placebo tablets for 5 to 7 days each. The placebo and selegiline tablets were matched to maintain the single-blind condition; however, for subject safety, study staff knew that placebo preceded selegiline.

Test doses of $20 \mathrm{mg}$ and $40 \mathrm{mg}$ of cocaine hydrochloride ( $1 \mathrm{mg}$ cocaine $/ 1 \mathrm{ml}$ saline) administered at $1 \mathrm{mg} / \mathrm{s}$ IV (i.e., over 20 and 40 seconds, respectively) were given on the first and second study day, respectively. Saline infusion was given as a control for each of the test doses of cocaine, the order of saline and cocaine was random, and the infusions were administered in a single-blind fashion approximately $50 \mathrm{~min}$ apart. These test doses were administered to ensure that subjects could safely tolerate cocaine in this setting and to confirm that the 40-mg cocaine dose resulted in subjective effects.

Subjects not excluded during the test dose administration sessions entered the assessment phase of the protocol $48 \mathrm{~h}$ later, when they underwent the first FDG-PET scan after receiving a placebo and radiotracer infusion. The following day, subjects received $40 \mathrm{mg}$ cocaine hydrochloride and radiotracer infusion prior to the second PET scan. This pair of PET scans (\#1 and \#2) was used to determine the effects of cocaine on cerebral glucose utilization. The night of the second PET scan, subjects were switched from nightly placebo pills to active medication (single-blind).

To determine whether subjects could safely tolerate cocaine infusion while taking selegiline, the test doses described above were repeated (subjects received the placebo injection coupled with test doses of $20 \mathrm{mg}$ and $40 \mathrm{mg}$ cocaine on sequential days) after they received 3 days of treatment with selegiline. Forty-eight hours later, the second pair of PET scans (\# 3 and \# 4) were performed on consecutive days in the same manner as the first pair (no-cocaine, then cocaine $40 \mathrm{mg}$ ) to determine the effects of cocaine on cerebral glucose utilization while under treatment with selegiline. Seven days of selegiline treatment results in virtually complete and irreversible MAO-B inhibition (Gerlach et al. 1996).

Determination of absolute glucose utilization could not be consistently obtained because arterial blood sampling was not attempted for safety reasons (four arterial catheters within 1 week), and venous access (for arterialized venous sampling) was not consistently obtained in this population with prior IV drug use experience because of vasospasm/clotting of the venous access, which occurred intermittently during the blood sampling period following the IV cocaine infusion. Therefore, for the statistical analysis, all region of interest (ROI) data were normalized to whole brain counts.

Before, during, and after each saline or cocaine infusion, subjects' physiologic (blood pressure and EKG) and subjective responses were closely monitored. A physician was present during the cocaine infusion for medical monitoring and for 1.5 hours thereafter. During the first two test IV cocaine infusions, adverse reactions (cardiovascular changes outside predetermined safety parameters) occurred in six subjects. When this occurred, the infusion was halted, appropriate medical intervention was instituted, and the subject was discontinued from the study.

PET brain image acquisition was performed $45 \mathrm{~min}$ after injection of the radioisotope and cocaine or saline injection. During the uptake period, the subjects performed a visual mental task. This continuous performance task (CPT) required that the subjects press a button when an $X$ was presented visually within a series of other letters. The CPT was performed to minimize irrelevant functional brain differences between occasions by maintaining alertness and attention, and minimizing distractions. In addition, subjects were instructed to focus on the CPT. The CPT was briefly interrupted at 3 , 10, and $30 \mathrm{~min}$ after cocaine or saline infusion to ascertain the subjective responses to cocaine. Subjects vocally rated on a 1 to 10 scale the extent to which they felt "high," depressed, sleepy, anxious, hungry, or had a desire for cocaine. The entire procedure, including CPT and subjective rating responses, was also performed during the initial test dose infusions of 20- and 40-mg cocaine. Thus, by the time of the first PET scan, these procedures were thoroughly familiar to the subjects.

Several hours after the final study dose of cocaine, subjects were discharged from the hospital if their mental status and vital signs were at baseline. Subjects were asked to return twice for follow-up at 1 and 2 weeks af- 
ter completion of the protocol to ascertain that no adverse events had occurred. None were reported.

\section{Imaging Procedures}

MRI Protocol. To quantify relative glucose utilization of small regions of interest, such as the hippocampus and amygdala, all subjects underwent a high resolution MRI using a Picker 1.5 Tesla instrument. The MRI was performed before the first test infusions to exclude patients with severe brain disease or increased ventricular brain ratio (VBR $>1: 10$ ), which may be associated with reduced response to cocaine infusion (Morgan et al. 1993). At the time of the MRI scan, each patient had a head/facemold constructed to reposition his or her head identically during the MRI and all subsequent PET scans, thus simplifying the coregistration of the MRI and multiple PET scans (Figure 1).

Before the MRI scan, the patient was positioned in a customized headrest that locked into both the MRI and PET headrests, and a rigid mold of the back of the patient's head was produced. The headmold was comfortable as long as the back of the head rested in the correct position, and all scans (MRI and PET) were performed while the patient was resting in this headmold and customized headrest apparatus. A facemold was also created of a warmed plastic mesh that conformed to the subject's facial contours and hardened as it cooled to room temperature. The facemold hardened while latched on to the customized headrest and, together with the headmold, formed a complete mold of the entire head that was used to reposition the head in all three coordinates before each of the subsequent PET scans.

Coronal and sagittal MRI pilot sequences were obtained to specify the location and spatial orientation of the head and the position of the axial image acquisition grid (Bartzokis et al. 1993; Bartzokis et al. 1994). All MRI data were derived from the subsequent two axial se- quences. Both sequences used two repetitions, $256 \times$ 192 view matrix, and contiguous slices. The first axial sequence was used to provide PET/MRI coregistration and provided excellent gray/white matter contrast. This sequence used a three-dimensional Fourier transform (3DFT) SPGR (spoiled-gradient-recalled) acquisition (TR $=30 \mathrm{~ms}, \mathrm{TE}=7 \mathrm{~ms}, 30^{\circ}$ angle) acquiring $1.5-\mathrm{mm}$ thick slices. The $\mathrm{T}_{1}$-weighted 3-D images have excellent gray-white contrast, making it possible to identify the alveus, the white matter tract that separates the hippocampus from the amygdala (Figure 1). The second axial sequence was used to obtain $\mathrm{T}_{2}$-weighted images using a transverse asymmetric dual spin-echo Carr-PurcellMeiboom-Gill sequence $(\mathrm{TR}=2500, \mathrm{TE}=20,90)$ that acquires interleaved 3-mm thick slices. The high CSFbrain contrast in these images makes measurements of ventricular size extremely accurate (Bartzokis et al. 1993).

PET Protocol. Subjects consumed only water after midnight before each PET scan and were not allowed to smoke for $2 \mathrm{~h}$ before the FDG injection and until the PET scan was complete. A Siemens 953/31 PET scanner that acquired $313.5-\mathrm{mm}$ slices simultaneously with an in-plane resolution of approximately $5-\mathrm{mm}$ full width at half maximum (FWHM) was used. The PET scanner and the well counter were cross-calibrated daily using phantoms containing a radionuclide. The attenuation correction was applied by finding the contours of the brain from the sinograms and empirically accounting for the attenuation of the skull and brain. Reconstruction was performed by standard filtered back-projection techniques.

The head/facemold was used to position the patient's head for each PET scan. Between 9:30 and 11:00 a.m., $40 \mathrm{ml}$ normal saline or $40 \mathrm{mg}$ of cocaine hydrochloride in $40 \mathrm{ml}$ of normal saline solution was infused
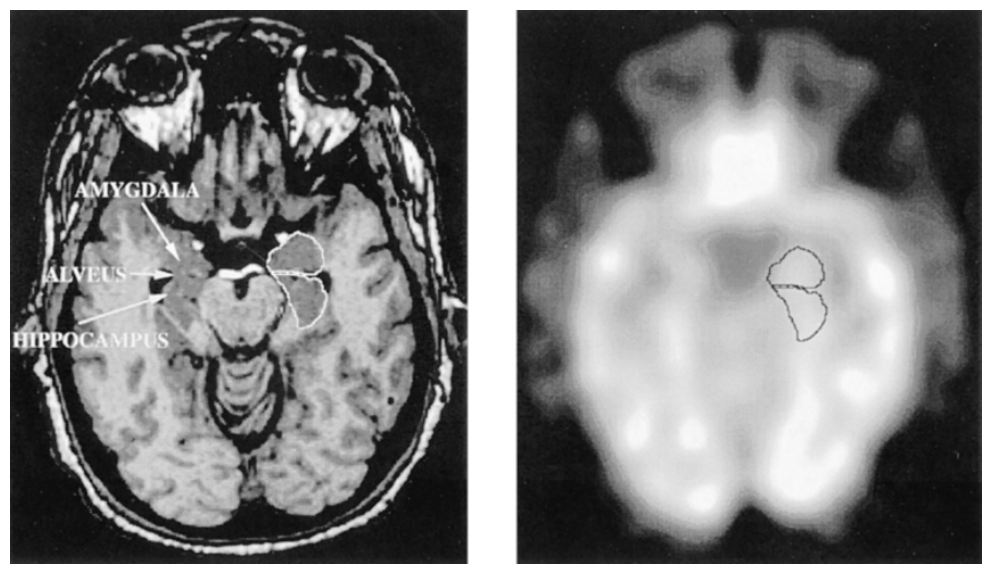

Figure 1. Demonstration of MRI/PET coregistration. The contours of the amygdala and hippocampus were manually drawn on the MRI images (left). The ROIs were then "transferred" onto the PET image, and superimposition was based on the best match of the MRI gray matter regions with the high activity gray matter regions of the PET image. 
into an arm vein over $40 \mathrm{~s}$, followed within $8 \mathrm{~s}$ by the FDG injection (approximately $5 \mathrm{mCi}$ ) and a $10 \mathrm{ml}$ saline flush.

Image Analysis. Imaging measures were obtained using a Macintosh configured image analysis workstation that read compact disks containing the original MRI and PET data stored in digital format. Data from MRI and PET scans were analyzed using an image analysis system (Evergreen Technologies, Castine, Maine) modified for MRI and PET scan analysis. The contours of the ROIs were drawn on the MRI images manually by the rater (JAF) (Bartzokis et al. 1993). The ROIs were then "transferred" onto the PET image, and superimposition was based on the best match of the MRI gray matter regions with the high activity gray matter regions of the PET image. The method is demonstrated in Figure 1 for the hippocampus and amygdala analysis.

\section{Data Analysis}

The effects of selegiline treatment and cocaine infusion on self-ratings of the experience of being "high" in each session were evaluated statistically in a 2 (selegiline) $\times 2$ (cocaine) within-subject factorial design. The analysis was performed using the general linear mixed model with repeated measures (SAS Proc MIXED, Littell et al. 1996). A series of similar analyses were performed to evaluate the effects of the experimental factors on regional glucose utilization within each of the three regions of interest (amygdala, hippocampus, thalamus). Although no a priori hypotheses were posited regarding the thalamus, this region was also included to examine the specificity of the limbic findings. The mixed model analyses involving PET measures were done separately within each brain region, including whole brain counts as a within-cell covariate. Compound symmetry was specified for the covariance structure of repeated measures, and the Satterthwaite option was used to estimate degrees of freedom for the statistical tests (Littell et al. 1996). Finally, Pearson correlations were computed within each of the two experimental sessions involving cocaine infusion to evaluate the association between PET measures and self-rated "high" (partialling whole brain counts).

\section{RESULTS}

Cocaine infusion produced maximal increases in the subjective rating of euphoria ("high") at $10 \mathrm{~min}$ after infusion both before $(\mathrm{t}=7.76, \mathrm{df}=21, p=.0001)$ and after $(\mathrm{t}=3.98, \mathrm{df}=21, p=.0007)$ selegiline treatment. After 7 days of selegiline treatment, the magnitude of the subjective high produced by cocaine infusion was reduced by $40 \%$, resulting in a significant cocaine by selegiline interaction $(\mathrm{F}=7.15, \mathrm{~d}=1,21, p=.014)$ (Figure 2).

Selegiline treatment also significantly altered normalized glucose utilization in the two limbic regions, but not in the thalamus. The results are summarized in Table 1. In the amygdala, the effects of cocaine infusion on glucose utilization differed, depending upon whether or not patients were being treated with selegiline (cocaine by selegiline interaction $\mathrm{F}=4.67, \mathrm{df}=1,19.8, p=$ .043; Figure 3). Before selegiline treatment, cocaine decreased glucose utilization in the amygdala, $(\mathrm{t}=1.85$, $\mathrm{df}=20.2, p=.079)$; after selegiline treatment, cocaine infusion was associated with a (nonsignificant) increase in glucose utilization in that region $(\mathrm{t}=1.13, \mathrm{df}=19.8$, $p=.27)$. In the hippocampus, a different effect was observed: selegiline treatment decreased normalized met-

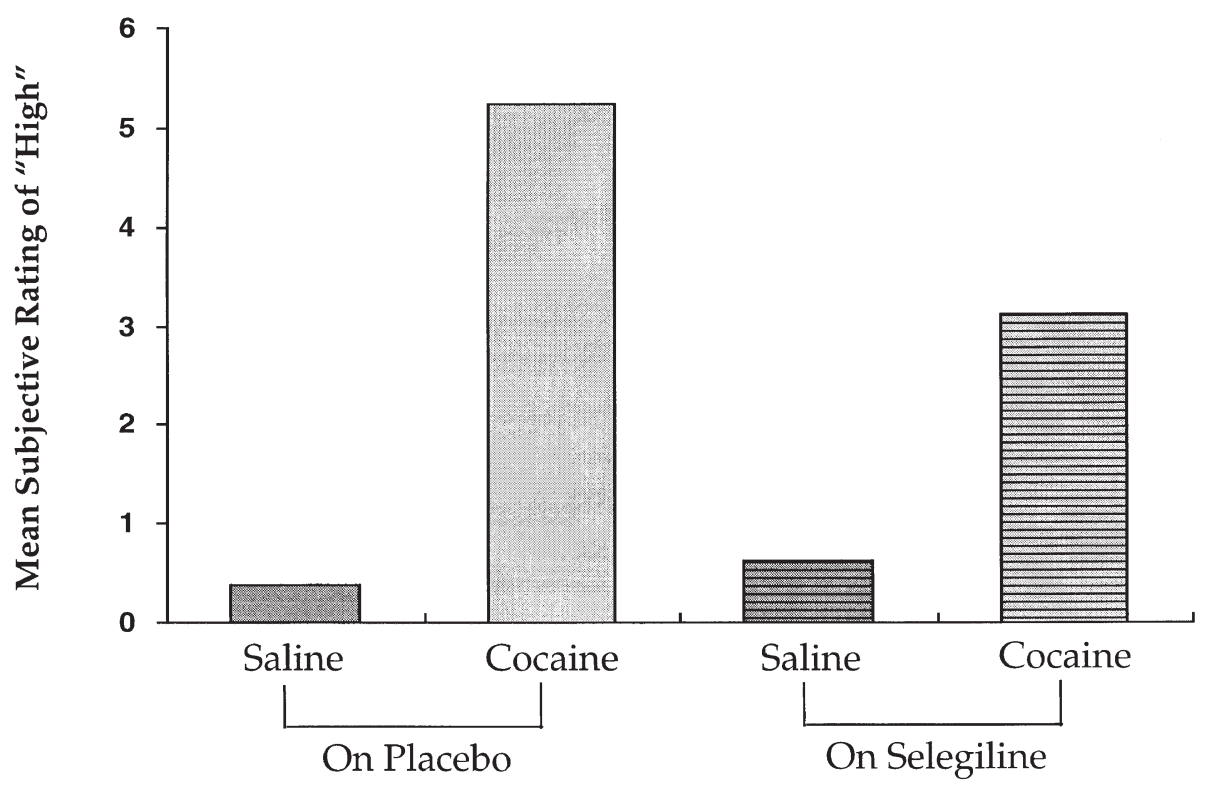

Figure 2. Subjective ratings of euphoria ("high") at $10 \mathrm{~min}$ after placebo and cocaine infusion (cocaine/selegiline interaction: $\mathrm{F}=$ 7.15, $p=.014[n=8, \mathrm{df}=1,21])$. Cocaine infusion produced a significant increase in the subjective "high". After 7 days of selegiline treatment, the magnitude of the subjective "high" produced by cocaine infusion was reduced by $40 \%$, resulting in a significant cocaine by selegiline interaction. 
Table 1. PET Measures of Metabolism in Selected Brain Regions (Means and Summary of ANCOVA Results $(2 \times 2$ Factorial $)$

\begin{tabular}{|c|c|c|c|c|c|c|c|}
\hline \multirow[b]{2}{*}{ Region } & \multicolumn{4}{|c|}{ Adjusted Means* } & \multicolumn{3}{|c|}{$\begin{array}{c}\text { ANCOVA } \\
p \text {-values }\end{array}$} \\
\hline & PET\#1 & PET\#2 & PET\#3 & PET\#4 & S & $\mathrm{C}$ & $\mathrm{S}^{*} \mathrm{C}$ \\
\hline Amygdala $^{a}$ & 54.26 & 50.35 & 49.33 & 51.64 & NS & NS & .04 \\
\hline Hippocampus ${ }^{b}$ & 83.85 & 83.42 & 80.27 & 81.35 & .01 & NS & NS \\
\hline Thalamus $^{c}$ & 215.96 & 217.73 & 212.37 & 218.65 & NS & NS & NS \\
\hline
\end{tabular}

${ }^{a}$ Selegiline* Cocaine Interaction: $\mathrm{F}=4.67 ; p=.043 ; \mathrm{df}=1,19.8$; pooled standard error $=3.5$.

${ }^{b}$ Selegiline main effect: $\mathrm{F}=7.70 ; p=.012 ; \mathrm{df}=1,20$; pooled standard error $=6.1$.

${ }^{c}$ No significant effects; pooled standard error $=7.4$.

${ }^{*}$ All analyses controlled for whole brain counts; $n=8$.

abolic activity in the hippocampus, whether cocaine was present or not (main effect $\mathrm{F}=7.70, \mathrm{df}=1,20, p=$ .012) (Figure 4).

\section{DISCUSSION}

Selegiline treatment significantly reduced the reinforcing "high" experienced during cocaine infusion. The PET data suggest that the reduction in this subjective response to cocaine by selegiline may involve altering the effect of cocaine on amygdala metabolism and reducing hippocampal metabolic activity. This supposition is consistent with findings from some animal studies (Lyons et al. 1996; Madras and Kaufman 1994; Pontieri et al. 1995; Stein and Fuller 1993; Imperato et al. 1993a; Hsu et al. 1996; Wilson et al. 1994; Whitelaw et al. 1996). These changes seemed to be specific to the limbic region, because they were not present in the thalamus control region.

The study has two principal limitations. First, the inability to obtain absolute metabolic rates reliably made it necessary to normalize regional data to whole brain counts. Although, as hypothesized, only the limbic regions showed significant effects (the thalamus control region did not), the normalized data cannot provide definitive information on the direction of the changes observed. This makes conclusions regarding the underlying mechanisms difficult. Second, the experiment was not randomized. Selegiline treatment always followed the placebo period. It is possible that general trends over time might have occurred, particularly trends associated with cocaine withdrawal. Arguing against this supposition is the fact that several cocaine infusions were done during the study period, thus ameliorating any generalized changes in brain metabolism related to withdrawal. Although subjects were not using their usual doses of cocaine during the project, they received a total of $200 \mathrm{mg}$ of cocaine over the 2-week period of the study. This cocaine exposure reduced the likelihood of global decreases in cerebral glucose utilization as a function of withdrawal (Volkow et al. 1991) and increased our confidence that the effects were attributed to selegiline and not artifacts attributable to the passage of time. All regional PET data were normalized to whole brain counts, which should also have reflected any global trends (Volkow et al. 1991). The thalamus control region did not demonstrate the changes observed in the limbic areas, indicating that the changes observed had anatomic specificity. Finally, withdrawal effects alone are unlikely to account for the reduction in the subjective euphoria produced by cocaine infusion. However, it is hard to know how much of the $40 \%$ re-

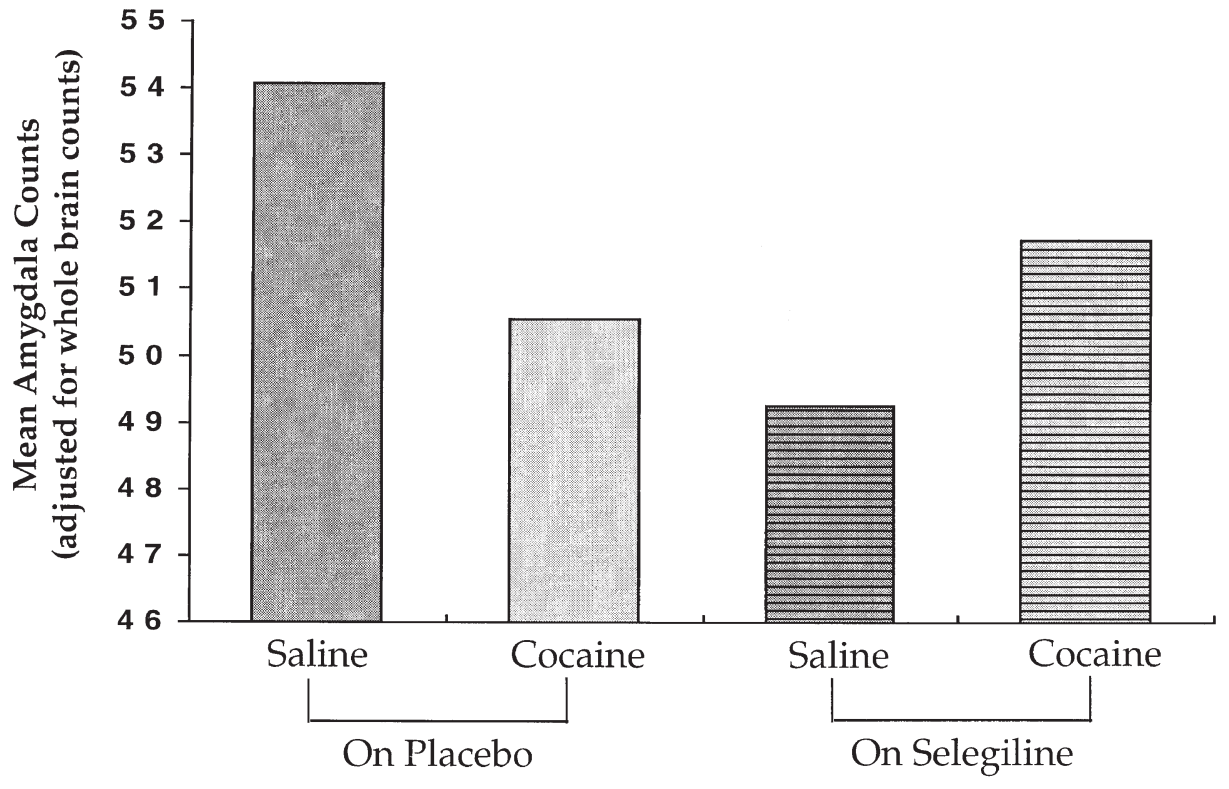

Figure 3. Cocaine and selegiline effects on the metabolism of the amygdala (cocaine/selegiline interaction: $\mathrm{F}=4.67, p=$ .043 , [ $n=8$, df $=1,19.8]$ ). Normalized glucose utilization in the amygdala. Before selegiline treatment, cocaine nonsignificantly decreased glucose utilization in the amygdala; whereas, after selegiline treatment, cocaine increased glucose utilization, resulting in a significant cocaine by selegiline interaction. 


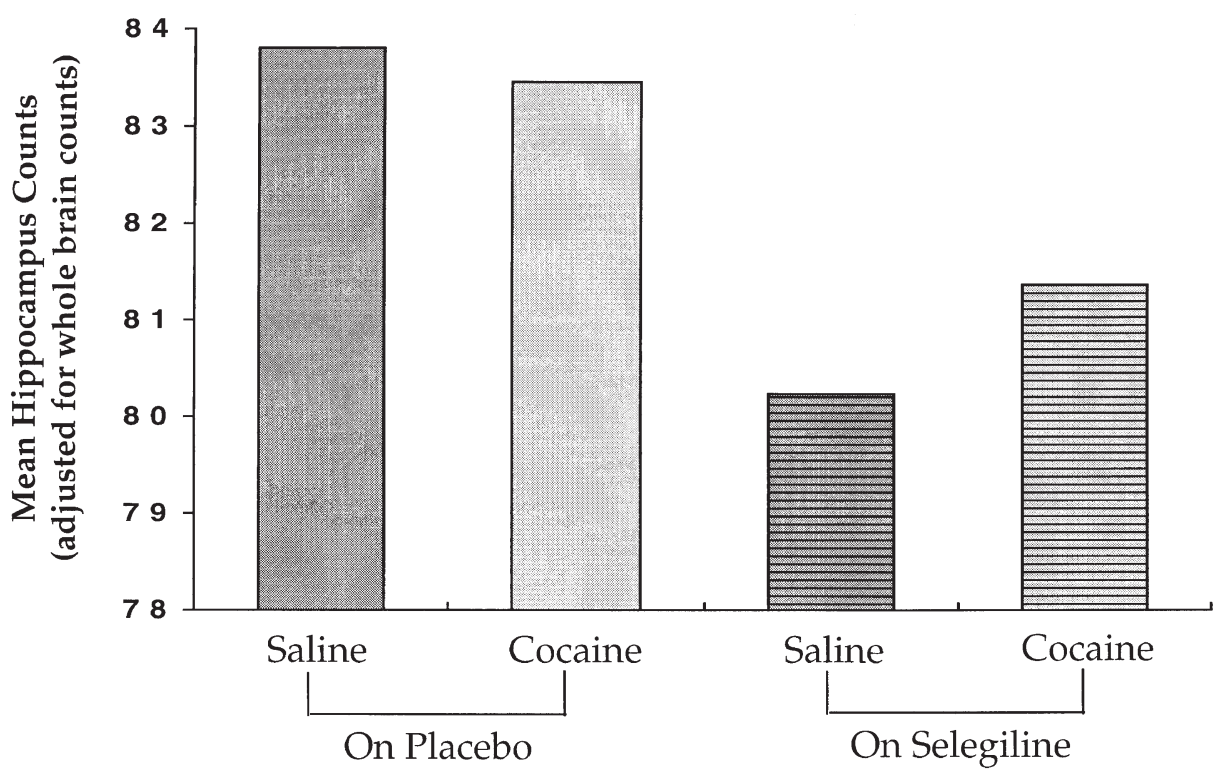

Figure 4. Cocaine and selegiline effects on the metabolism of the hippocampus (selegiline effect: $\mathrm{F}=7.70, p=.012[n=8$, $\mathrm{df}=1,20]$ ). Normalized glucose utilization in the hippocampus. Selegiline treatment decreased normalized metabolic activity in the hippocampus whether cocaine was present or not, resulting in a significant main effect for selegiline. duction in euphoria can be attributed to selegiline or to such other effects as withdrawal or tolerance to the effects of cocaine.

The amygdala is involved in motivated behavior (Aggleton 1992; Gaffan 1992), and amygdala response to cocaine is probably more closely linked to cocaine's reinforcing effects than to direct pharmacological effects of the drug. There is no amygdala response to cocaine, for example, in cocaine-naive nonhuman primates (Lyons et al. 1996); whereas, increased firing of amygdala neurons was shown in response to stimuli that had repeatedly been paired with positive reinforcers (Nishijo et al. 1988). Rats that self-administered cocaine showed increased dopamine and serotonin levels in the amygdala, but yoked rats who received equal amounts on what amounted to random schedules did not (Wilson et al. 1994). These animal studies are consistent with human data indicating an essential role for the amygdala in the coupling of sensory stimuli with affect and the establishment of sensory-affect associations underlying emotional conditioning (Bechara et al. 1995). In the current sample of cocaine-experienced addicts, amygdala responses to cocaine after selegiline treatment were attenuated, and this may interfere with cocaine's emotional reinforcing properties.

The hippocampus is central to declarative memory functions (Bechara et al. 1995). The observation that psychostimulants (cocaine and amphetamines) as well as opiates produce especially marked dopamine-dependent increases in hippocampus acetylcholine release lead to speculation that this dopamine-dependent mechanism may play a role in establishing memories of the rewarding effects of drugs (Imperato et al. 1993a; Imperato et al. 1993b; Imperato et al. 1996). Conditioned responses and the memory circuits involved may be pertinent to human cocaine dependence (White 1996). Conditioned, cue-induced, cocaine craving, triggered by memories of euphoria associated with cocaine use, is sometimes a part of the relapse process (White 1996; Childress et al. 1995; Grant et al. 1996). Recent PET studies have shown widespread activation of memory circuits in cocaine addicts who were experiencing cueinduced cocaine craving as a result of exposure to visual and tactile cues or receiving cocaine infusions while undergoing functional magnetic resonance imaging (Grant et al. 1996; Breiter et al. 1997). In the current study, selegiline treatment significantly altered metabolic activity in the hippocampus and may interfere with the conditioned effects of cocaine.

Selegiline may be a useful pharmacologic adjunct in the treatment of cocaine addiction. In an open label study, the nonselective MAO inhibitor phenelzine decreased cocaine use in outpatients, but its utility may be limited by the reported abuse liability and possible adverse cardiovascular interactions (Golwyn 1988). Selegiline has neither of these two limitations (Schneider et al. 1994; Haberny et al. 1995). Treatment of addictions with other dopamine agonists that have higher abuse potentials has also been reported by relapsing addicts to reduce reinforcing subjective effects (Rothman 1995; Rothman et al. 1996). The current PET findings suggest that selegiline may reduce the reinforcing subjective effects of cocaine by altering limbic function. Selegiline could, therefore, minimize the reinforcing effects of occasional limited use of cocaine or "slips," which often precede relapses, and thus reduce the chances of fullblown relapse. A similar reduction of alcohol's reinforcing subjective effects has been postulated to underlie naltrexone's effectiveness in the treatment of alcohol dependence (O’Brien et al. 1996; Volpicelli et al. 1995). 
Data from a recent multicenter double-blind, placebo-controlled, and parallel-group clinical trial evaluating the efficacy of a 10-mg daily dose of selegiline for cocaine dependence seem to support this hypothetical mechanism of action. Significant improvements in outcome measures were observed only in the subset of subjects who, after entering the study, continued using cocaine during the single-blind placebo "run in" first week of the study (Bridge et al. 1997). By reducing cocaine's subjective effects and, thus, minimizing its reinforcing action, selegiline could be particularly helpful for those patients who continue "slipping" in the early stages of their psychosocial treatment.

\section{ACKNOWLEDGMENTS}

This work was supported by the Medication Development Division of the National Institute on Drug Abuse (1YO1 DA 50038), the Research and Psychiatry Services of the Department of Veterans Affairs, the Marie Wilson Howells Endowment and by Pharmavene Inc., Gaithersburg, Maryland, who provided matched placebo and selegiline tablets. The authors thank Edythe D. London, Ph.D. of the NIDA Brain Imaging Center for consultations in designing the protocol and reviewing the manuscript; Nora Volkow, M.D. of the Brookhaven National Laboratory for consultations in designing the protocol; Charles Brown, M.D. for performing the PET scans; Horst Meissner, M.D., Michael Goldman, M.D., Sc.D., and Jill Tureaud, R.N. for medical screening and monitoring cocaine infusion; Judith Lindholm, M.S. for coordinating infusions and recruitment; and Marguerite Callinan, M.A. for coordinating the imaging sessions.

\section{REFERENCES}

Aggleton JP (1992): The functional effects of amygdala lesions in humans: A comparison with findings from monkeys. In Aggleton JP (ed), The Amygdala: Neurobiological Aspects of Emotions, Memory, and Mental Dysfunction. New York, Wiley, pp 485-504

Bartzokis G, Mintz J, Marx P, Osborn D, Gutkind D, Chiang F, Phelan CK, Marder SR (1993): Reliability of in vivo volume measures of hippocampus and other brain structures using MRI. Magn Reson Imaging 11:993-1006

Bartzokis G, Mintz J, Sultzer D, Marx P, Herzberg J, Phelan C, Marder S (1994): In vivo MR evaluation of agerelated increases in brain iron. AJNR 15:1129-1138

Bechara A, Tranel D, Damasio H, Adolphs R, Rockland C, Damasio AR (1995): Double dissociation of conditioning and declarative knowledge relative to amygdala and hippocampus in humans. Science 269:1115-1118

Berger SP, Hall S, Mickalian JD, Reid MS, Crawford CA, Delucchi K, Carr K (1996): Haloperidol antagonism of cue-elicited cocaine craving. Lancet 347:504-508

Blaine JD, Ling W (1992): Psychopharmacologic treatment of cocaine dependence. Psychopharmacol Bull 28:11-14

Breiter HC, Gollub RL, Weisskoff RM, Kennedy DN, Makris N, Berke JD, Goodman JM, Kantor HL, Gastfriend DR,
Riorden JP, Mathew RT, Rosen BR, Hyman SE (1997): Acute effects of cocaine on human brain activity and emotion. Neuron 19:591-611

Bridge TP, Weinreib R, Batki S, Bartzokis G, Li S-H, Hill J (1997): Selegiline treatment for cocaine dependence. Proceedings of American College of Neuropsychopharmacology 36th Annual Meeting. Scientific Abstracts, 207

Childress AR, Mozley PD, Fitzgerald J, Reivich M, Jaggy J, O'Brien CP (1995): Regional brain flow during cueinduced cocaine craving. NIDA Research Monograph 162:194

Gaffan D (1992): Amygdala and the memory of reward. In Aggleton JP (ed), The Amygdala: Neurobiological Aspects of Emotions, Memory, and Mental Dysfunction. New York, Wiley-Liss

Gerlach M, Youdim MBH, Riederer P (1996): Pharmacology of selegiline. Neurology 47:S137-S145

Golwyn DH (1988): Cocaine abuse treated with phenelzine. Int J Addict 23:897-905

Grant S, London ED, Newlin DB, Villemagne VL, Liu X, Contoreggi C, Phillips RL, Kimes AS, Margolin A (1996): Activation of memory circuits during cue-elicited cocaine craving. Proc Natl Acad Sci USA 93:1204012045

Haberny KA, Walsh SL, Ginn DH, Wilkins JN, Garner JE, Setoda D, Bigelow GE (1995): Absence of acute cocaine interactions with the MAO-B inhibitor selegiline. Drug Alcohol Depend 39:55-62

Hsu KS, Huang CC, Su MT, Tsai JJ (1996): L-deprenyl (selegiline) decreases excitatory synaptic transmission in the rat hippocampus via a dopaminergic mechanism. J Pharmacol Exper Therapeut 279:740-747

Imperato A, Obinu MC, Gessa GL (1993a): Stimulation of both D1 and D2 receptors facilitates in vivo acetocholine release in the hippocampus. Brain Res 618:341-345

Imperato A, Obinu MC, Gessa GL (1993b): Effects of cocaine and amphetamine on acetylcholine release in the hippocampus and caudate nucleus. Eur J Pharmacol 238:377-381

Imperato A, Obinu MC, Casu MA, Mascia MS, Carta G, Gessa GL (1996): Chronic morphine increases hippocampal acetylcholine release: Possible relevance in drug dependence. Eur J Pharmacol 302:21-26

Knoll J, Miklya I, Knoll B, Marko R, Kelemen K (1996): (-)Deprenyl and (-)1-phenyl-2-propylaminopentane, [(-)PPAP], act primarily as potent stimulants of action potentialtransmitter release coupling in the catecholaminergic neurons. Life Sci 58:817-827

Koob GF, Weiss F (1992): Neuropharmacology of cocaine and ethanol dependence. In Galanter $M$ (ed), Recent Developments in Alcoholism, vol. 10. New York, Plenum Press

Littell RC, Milliken GA, Stroup WW, Wolfinger RD (1996): SAS System for Mixed Models. Cary, NC, SAS Institute, Inc., p 633

London ED, Cascella NG, Wong DF, Philips RL, Dannals RF, Links JM, Herning R, Grayson R, Jaffe JH, Wagner HN, Jr (1990): Cocaine-induced reduction of glucose utilization in human brain. Arch Gen Psychiat 47:567-574

Lyons D, Freidman DP, Nadar MA, Porrino LJ (1996): 
Cocaine alters cerebral metabolism within the ventral striatum and limbic cortex of monkeys. J Neurosci 16:1230-1238

Madras BK, Kaufman MJ (1994): Cocaine accumulates in dopamine-rich regions of primate brain after I.V. administration: Comparison with mazindol distribution. Synapse 18:261-275

Morgan MJ, Cascella NG, Stapleton JM, Phillips RL, Yung BCK, Wong DF, Shaya EK, London ED (1993): Sensitivity to subjective effects of cocaine in drug abusers: Relationship to cerebral ventricle size. Am J Psychiat 150:1712-1717

Nishijo H, Ono T, Nishino H (1988): Single neuron responses in amygdala of alert monkey during complex sensory stimulation with affective significance. J Neurosci 10:3570-3583

O'Brien CP, Volpicelli LA, Volpicelli JR (1996): Naltrexone in the treatment of alcoholism: A clinical review. Alcohol 13:35-39

Paterson IA, Davis BA, Durden DA, Juorio AV, Yu PH, Ivy G, Milgram W, Mendonca A, Wu P, Boulton AA (1995): Inhibition of MAO-B by (-)-deprenyl alters dopamine metabolism in the Macaque (Macaca facicularis) brain. Neurochem Res 20:1503-1510

Pearson GD, Jeffery PJ, Harris GJ, Ross CA, Fischman MW, Camargo EE (1993): Correlation of acute cocaineinduced changes in local cerebral blood flow with subjective effects. Am J Psychiat 150:495-497

Pontieri FE, Mainero C, La Riccia M, Passarelli F, Orzi F (1995): Functional correlates of repeated administration of cocaine and apomorphine in the rat. Eur J Pharmacol 284:205-209

Rothman RB (1995): Treatment of alcohol and cocaine addiction by the combination of pemoline and fenfluramine: A preliminary case series (letter). J Sub Abuse Treatm 12:449-453

Rothman RB, Ayestas M, Baumann MH (1996): Phentermine pretreatment antagonizes the cocaine-induced rise in mesolimbic dopamine. Neuroreport 8:7-9

Satel SL, Krystal JH, Delgado PL, Kosten TR, Charney DS (1995): Tryptophan depletion and attenuation of cueinduced craving for cocaine. Am J Psychiat 152:778-783

Schneider LS, Tariot PN, Goldstein B (1994): Therapy with l-deprenyl (selegiline) and relation to abuse liability. Clin Pharmacol Therapeut 56:750-756

Schultz W, Apicella P, Ljungberg T, Romo R, Scarnati E (1993): Reward-related activity in the monkey striatum and substantia nigra. Prog Brain Res 99:227-235

Stein EA, Fuller SA (1993): Cocaine's time action profile on regional cerebral blood flow in the rat. Brain Res 626:117-126

Tutton CS, Crayton JW (1993): Current pharmacotherapies for cocaine abuse: A review. J Addict Dis 12:109-127

Volkow ND, Fowler JS, Wolf AP, Hitzemann R, Dewey S, Bendriem B, Alpert R, Hoff A (1991): Changes in brain glucose metabolism in cocaine dependence and withdrawal. Am J Psychiat 148:621-626

Volpicelli JR, Watson NT, King AC, Sherman CE, O'Brien CP (1995): Effect of naltrexone on alcohol "high" in alcoholics. Am J Psychiat 152:613-615

White NM (1996): Addictive drugs as reinforcers: Multiple partial actions on memory systems. Addiction 91:921949

Whitelaw RB, Markou A, Robbins TW, Everitt BJ (1996): Excitotoxic lesions of the basolateral amygdala impair the acquisition of cocaine-seeking behaviour under a second-order schedule of reinforcement. Psychopharmacology 127:213-224

Wilson JM, Nobrega JN, Corrigall WA, Coen KM, Shannak K, Kish SJ (1994): Amygdala dopamine levels are markedly elevated after self- but not passive-administration of cocaine. Brain Res 668:39-45

Wise RA (1996): Neurobiology of addiction. Curr Opinion Neurobiol 6:243-251 